\title{
CARDIOPULMONARY EXERCISE TESTING RESPONSES AND PRO-BNP VALUES IN ADULTS WITH MILD DEGREES OF DIASTOLIC DYSFUNCTION
}

\author{
H.R. Ahmadian ${ }^{1}$, J.R. Sherratt' ${ }^{2}$ K.M. Lochner ${ }^{1}$, M. Dubois ${ }^{1}$, K.M. Leclerc ${ }^{1}$
}

\begin{abstract}
Diastolic dysfunction (DD) can range from a process of normal aging to overt heart failure (HF). Cardiopulmonary exercise testing (CPET) variables (VO2max and VE/VCO2 slope), and pro-BNP values are abnormal in diastolic HF patients. These CPET parameters have not previously been investigated in adults with varying degrees of DD without clinical HF. Ten subjects with grade 1, and twelve with grade 2 DD had pro-BNP values measured, and subseqeuntly underwent CPET assessment. No subjects with grade 3 or $4 \mathrm{DD}$ were eligible for enrollment based on pre-existing HF or co-morbidities. CPET responses were normal for all individuals and without significant differences between grade I and II DD. Pro-BNP values were significantly higher in those with grade II dysfunction but still under a threshold consistent with HF. We conclude that adults with grade 1 or 2 DD have normal and prognostically favorable CPET responses and pro-BNP values.
\end{abstract}

Key words: Diastolic dysfunction, cardiopulmonary exercise testing, oxygen consumption, VE/VCO2 slope, natriuretic peptide.

\section{Introduction}

Impaired left ventricular diastolic function is common and can develop asymptomatically as a consequence of normal aging. However, in advanced stages it can manifest as overt heart failure with preserved ejection fraction (HFpEF) (1).

Cardiopulmonary exercise testing (CPET) enables integrative assessment of cardiopulmonary responses to exercise. Maximum oxygen consumption (VO2max) and the ventilatory equivalent of carbon dioxide (VE/VCO2) slope have been demonstrated to be abnormal in those with HFpEF (2-4). Whether CPET abnormalities will manifest at various stages of diastolic dysfunction in the absence of clinically diagnosed heart failure has not been previously investigated.

Pro-BNP (B-type natriuretic peptide) is a neurohormone released by the myocardium in response to increased ventricular volume or pressures and has been shown to be elevated in patients with advanced diastolic dysfunction or HFpEF (5).

The aim of this study was to determine to what extent a degree of diastolic dysfunction reveals abnormalities, if any, of VO2max, VE/VCO2 slope, and pro-BNP in adults

1. Department of Cardiology; 2. Department of Pulmonary and Critical Care Medicine, San Antonio Military Medical Center, San Antonio, Texas.

Corresponding Author: Kenneth Leclerc, Brooke Army Medical Center, Department of Cardiology. 3551 Roger Brooke Dr. San Antonio, TX 78234, Phone: 210-9165145, Fax: 210-916-3051, kenneth.m.leclerc.mil@mail.mil without clinical HFpEF.

\section{Methods}

All procedures and protocols were approved by the San Antonio Military Medical Center Institutional Review Board. All of the authors have no conflicts to disclose and received no payment for this study. Signed consent was obtained for all participants.

Eligible subjects were 18-85 years of age with ejection fraction $>50 \%$, no worse than moderate valvular heart disease, and able to safely perform treadmill exercise. Echocardiograms and medical records were reviewed and grade of diastolic dysfunction was determined using criteria published by the American Society of Echocardiography (6).

Ten subjects with Grade I and twelve subjects with grade II diastolic dysfunction were enrolled. Our intent had been to enroll candidates with more advanced diastolic dysfunction (grades III and IV) but they were found to have either established HFpEF or prohibitive co-morbidities and were unable to be enrolled.

Patients underwent treadmill CPET and a serum pro-BNP was drawn no more than 24 hours prior to the CPET. Standard endpoints for cessation of the exercise test were utilized. V02 peak was the highest value recorded. It was considered to be prognostically poor at a value $<14 \mathrm{ml} / \mathrm{kg} / \mathrm{min} 2$. The VE/VCO2 slope was defined as the best-fit slope of all data points (7). 
A slope $>34$ is accepted as an abnormally high value that portends poorer prognosis. A pro-BNP value of $>300 \mathrm{pg} / \mathrm{ml}$ was considered to be abnormal across all age groups to a degree that could be consistent with congestive heart failure $(5,8)$.

\section{Results}

Subjects were demographically similar. Only one grade I subject and 6 of 12 grade II subjects were on beta blockers. None had a clinical history of heart failure (Table 1).

Table 2 displays selected test data. Maximal exercise was achieved in all subjects. Lateral mitral annulus $\mathrm{E} / \mathrm{e}^{\prime}$ ratio and left atrial volume index (LAVI), both echocardiographic reflections of elevated left atrial pressure and/or advanced diastolic dysfunction, were not, on average, elevated within each group nor significantly different between the two groups. Only one grade I subject, and two grade II subjects had elevated $\mathrm{E} / \mathrm{e}^{\prime}$ ratios. Three subjects in the grade I diastolic dysfunction group had a LAVI exceeding the upper limit of normal value of 28 , as did four of the grade II subjects.

All of the grade I subjects had a pro-BNP values well within normal, all being less than $76.5 \mathrm{pg} / \mathrm{ml}$. ProBNP was significantly higher in the group with grade II diastolic dysfunction (34.8 vs. $263.3 \mathrm{pg} / \mathrm{ml} ; \mathrm{p}=.003$ ), driven primarily by two outlying values (703 and 1260 $\mathrm{pg} / \mathrm{ml}$ ). If these two values were excluded there was a non-significant trend for elevated pro-BNP in the grade II diastolic dysfunction group. (average pro-BNP of 119.5 vs. $34.8 \mathrm{pg} / \mathrm{ml}$ ).

In all subjects, peak VO2 was well above the conventional prognostically poor value of $14 \mathrm{ml} / \mathrm{kg} /$ min. There was no significant difference between the two groups. In subjects with grade I diastolic dysfunction,

Table 1

Baseline characteristics of subjects by degree of diastolic dysfunction

\begin{tabular}{llll}
\hline Variable & $\begin{array}{l}\text { Grade I }(\mathbf{n}=\mathbf{1 0}) \\
\text { Average (range) }\end{array}$ & $\begin{array}{l}\text { Grade II (n=12) } \\
\text { Average (range) }\end{array}$ & P value \\
\hline Gender & 9 male, 1 female & 8 male, 4 female & 0,323 \\
Age (years) & $65.6(51.0-84.0)$ & $61.6(53.0-74.0)$ & 0,426 \\
Weight (kg) & $92.6(77.1-154.2)$ & $98.4(60.0-11.4)$ & 0,56 \\
$\begin{array}{l}\text { Body Mass Index } \\
\text { (BMI) }\end{array}$ & $31.5(26.1-43.7)$ & $30.6(23.3-40.0)$ & 0,703 \\
Race & & & 0,457 \\
$\begin{array}{l}\text { African American } \\
\text { Caucasian }\end{array}$ & 3 & 2 & \\
Hispanic & 3 & 8 & 0,074 \\
B-Blocker use & 1 & 2 & \\
\hline
\end{tabular}

$\mathrm{VE} / \mathrm{VCO} 2$ slope was well within normal $(<30)$ in 8 of 10 subjects, with two slightly elevated values but none exceeding the prognostically poor threshold of 34. In grade II subjects, 7 of 12 had a slope $<30$. Of the remaining 5 , only one was greater than 34 . Overall, there was no statistical difference between groups.

\section{Discussion}

To our knowledge, the investigation of peak VO2, VE/ VCO2 slope, along with pro-BNP, has not been examined in varying degrees of diastolic dysfunction in the absence of clinical heart failure.

Our results suggest that in patients without clinical heart failure that have grade I or II diastolic dysfunction, selected CPET measures and pro-BNP are likely to be within normal range and prognostically favorable.

This is consistent with other published reports. Crowson, et al demonstrated higher BNP values in patients with rheumatoid arthritis, particularly those with advancing degrees of diastolic dysfunction as compared to normal subjects though none were of a value that would be diagnostic of congestive heart failure (> $100 \mathrm{pg} / \mathrm{ml}$ in the case of BNP) (9). The specifics of the echocardiographic classifications were not published in their manuscript. In a cross sectional study in a Portuguese community of individuals over the age of 45 years, Goncalves found an incidence of $32.9 \%$ with impaired relaxation (grade I diastolic dysfunction) by echocardiographic measures but there was no significant difference in BNP elevation (defined as $>30 \mathrm{pg} /$ $\mathrm{ml}$ ) compared to normal subjects (10). Romano et al examined BNP values in 127 adult outpatients with type II diabetes (11). Based on echocardiography, $42 \%$ had impaired relaxation (grade I diastolic dysfunction); the remainder had normal diastolic profiles. Similar to our results, the BNP values were well within normal and without significant differences between the two groups.

One provocative finding is worth specific highlight. One individual in the grade II diastolic dysfunction group had the highest VE/VCO2 slope (36.4), the highest pro-BNP value of $1260 \mathrm{pg} / \mathrm{mil}$, the highest left atrial volume index of $49.5 \mathrm{ml} / \mathrm{m} 2$, and lowest LVEF at $50 \%$. Within 6 months following this testing she was seen clinically with a new diagnosis of HFpEF based on development of lower extremity edema and progressive shortness of breath. Though only one case in point, it raises the consideration that the findings of abnormal VE/VCO2 slope, elevated pro-BNP, particularly in combination with other echocardiographic measures of advanced diastolic dysfunction, such as an elevated LAVI, may allow identification of individuals at risk for the development of clinical heart failure.

Our results suggest that in patients without clinical heart failure that have grade I or II diastolic dysfunction, selected CPET measures and pro-BNP are likely to be at prognostically good levels. Routine assessment by CPET 
Table 2

Selected CPET, Echo, and pBNP values by grade of diastolic dysfunction

\begin{tabular}{lccc}
\hline Variable & $\begin{array}{c}\text { Grade I (n= 10) } \\
\text { Average (range) }\end{array}$ & $\begin{array}{c}\text { Grade II (n-=12) } \\
\text { Average (range) }\end{array}$ & P value \\
\hline $\begin{array}{l}\text { peak VO2 (ml/kg/ } \\
\text { min) }\end{array}$ & $23.5(17-34.6)$ & $22.7(12.5-32.1)$ & 0,818 \\
$\%$ of max predicted & $101.5(68.0-128.0)$ & $95.8(83.0-120.0)$ & 0,431 \\
VE/VCO2 slope & $27.4(20.3-33.6)$ & $29.2(25.4-36.4)$ & 0,279 \\
RER & $1.16(1.05-1.25)$ & $1.15(1.00-1.29)$ & 0,863 \\
Pro-BNP & $34.8(5.6-76.4)$ & $263.2(27.6-$ & 0.003 \\
& $10.1(6.1-17.8)$ & $8.5(6.2-15.2)$ & 0.708 \\
$\begin{array}{l}\text { Lateral E/e' ratio } \\
\text { Left atrial volume } \\
\text { index }\end{array}$ & $25.0(17.2-34.7)$ & $25.3(18.9-49.7)$ & 0,269 \\
\hline
\end{tabular}

$\mathrm{VO} 2$ - Maximum oxygen consumption; VE/VCO2- ventilator equivalent of carbon dioxide; RER- respiratory exchange ratio; Lateral $\mathrm{E} / \mathrm{e}^{\prime}$ ratio: Ratio of early diastolic filling peak velocity from mitral inflows to mitral annulus early diastolic peak velocity from tissue Doppler.

or pro-BNP would be expected to be of low diagnostic yield. However, these negative results could serve as a point of reassurance for both the patient and clinician. In situations where clinical suspicion for possible heart failure is high, the finding of abnormal VE/VCO2 slope and/or elevated pro-BNP may be an early clue to the future development of clinical heart failure.

There are several limitations of this study. Subjects were clinically and ethnically diverse, precluding extrapolation to a single clinical entity, gender, or race. The majority of our subjects were men, limiting the extension of our conclusions to women.

The absence of suitable subjects with restrictive filling (grades III and IV) limited our overall enrollment to a smaller sample size than initially planned which restricts conclusions to those with lower grades of diastolic dysfunction.

It is possible for diastolic LV filling pressures to be somewhat dynamic (12). Determination of diastolic dysfunction by echocardiographic measures at different points of time could support a different rating (e.g. from grade I to grade II and vice versa) which would have altered subject classification in this study.

In summary, this was the first investigation of selected CPET parameters of prognostic significance and associated pro-BNP values in patients without heart failure, with grades I and II diastolic dysfunction.

Our results found that for both groups on average, peak VO2 and VE/VCO2 slope were within normal and in a prognostically favorable range. Pro-BNP was significantly higher in the grade II diastolic dysfunction group but in both groups as a whole, below a value consistent with congestive heart failure. In the vast majority of patients with these lesser degrees of diastolic dysfunction, assessment with CPET and pro-BNP measurement would provide reassuring negative results. In patients with a high suspicion for HFpEF, abnormal $\mathrm{VE} / \mathrm{VCO} 2$ slope by CPET, and/or elevated pro-BNP may serve as a clue for the progression to clinical heart failure and warrant closer follow-up. The provocative findings in this small investigative study promote future investigations on a larger scale.

\section{Acknowledgments: None}

\section{Funding: None}

Disclosure: The views expressed herein are those of the authors and do not reflect the official policy or position of the Department of the Army, Department of Defense, or the US Government. The authors are employees of the US government. This work was prepared as part of their official duties and, as such, there is no copyright to be transferred.

\section{References}

1. Klein AL and Asher CR. Diseases of the pericardium, restrictive cardiomyopathy, and diastolic dysfunction. In: Topol EJ ed. Textbook of Cardiovascular Medicine. Philidelphia: Lippincott Williams and Wilkins. pp 2007;420-58.

2. Mancini DM, Eisen H, Kussmaul W, Mull R, Edmunds LH Jr, Wilson JR. Value of peak exercise consumption for optimal timing of cardiac transplantation in ambulatory patients with heart failure. Circulation 1991;83:778-86.

3. Guazzi M, Myers J, Arena R. Cardiopulmonary exercise testing in the clinical and prognostic assessment of diastolic heart failure. J Am Coll Cardiol 2005;46:1883-90.

4. Arena R, Myers J, Guazzi M. The clinical and research applications of aerobic capacity and ventilatory efficiency in heart failure: an evidence based review. Heart Failure Rev 2008;13:245-69.

5. Hobbs RE . Using BNP to diagnose, manage, and treat heart failure. Cleve Clin J Med 2003;70:333-36.

6. Nagueh SF, Appleton CP, Gillebert CT, Marino PN, Oh JK, Smiseth OA, Waggoner AD, Flachskampf FA, Pellikka PA, Evangelista A. Recommendations for the evaluation of left ventricular diastolic function by echocardiography. J Am Soc Echocardiogr 2009;22:107-33.

7. Guazzi M, Adams V, Conraads V, Halle M, Mezzani A, Arena R, Fletcher GF, Forman DE, Kitzman DW, Lavie CJ, Myers J. EACPR/AHA Scientific Statement. Clinical recommendations for cardiopulmonary exercise testing data assessment in specific patient populations. Circulation 2012;126:2261-74.

8. Composite Health Care System (CHCS), San Antonio Military Medical Center, Lab Test Interpretation for proBNP. Accessed February 2012.

9. Crowson CS, Myasoedova E, Davis JM III, Roger VL, Karon BL, Borgeson D, Rodheffer RJ, Therneau TM, Gabriel SE. Use of B-type natriuretic peptide as a screening tool for left ventricular diastolic dysfunction in rheumatoid arthritis without clinical cardiovascular disease. Arthritis Care and Research 2011;63:729-34

10. Goncalves A, Almeida PB, Lourenco P, Alvelos M, Betrencourt P, Azevedo A. Clinical significance of impaired relaxation in middle-aged and elderly adults in the general population. Rev Port Cardiol 2010;29:1799-1806.

11. Romano S, Di Mauro M, Fratini S, Guarracini L, Guarracini F, Poccia G, Penco M. Early diagnosis of left ventricular diastolic dysfunction in diabetic patients: a possible role for natriuretic peptides. Cardiovascular Diabetol 2010;9:89.

12. Ogunyankin KO. Assessment of left ventricular diastolic dysfunction: the power, possibilities, and pitfalls of echocardiographic imaging techniques. Can J Cardiol 2011;27: 311-8. 